\title{
Haematological and biochemical reference intervals in adult Maine Coon cat blood donors
}

\author{
Eva Spada ${ }^{1}$, Maria Teresa Antognoni², Daniela Proverbio', \\ Elisabetta Ferro', Vittorio Mangili² and Arianna Miglio²
}

\begin{abstract}
Objectives The objectives of this study were to derive Maine Coon haematological and biochemical reference intervals (RIs) from adult healthy blood donors, to validate (or reject) the use of published RIs for the general feline population in this breed, and to evaluate the effects of age, sex and weight on the haematological and biochemical results.

Methods Haematological and biochemical data were retrieved retrospectively from a database of 81 healthy adult Maine Coon cat blood donors and were analysed to generate normal Rls. Rls were determined and compared with established non-breed-specific feline RIs according to the Clinical and Laboratory Standards Institute guidelines and the American Society of Veterinary Clinical Pathology guidelines using Reference Value-Advisor (version 2.1) software.

Results The age of the cats ranged from 1-8 years (mean 4.4 years), 42 were female and 39 were male, and weights ranged from 4.9-8.5 kg (mean $6.7 \mathrm{~kg}$ ). New Maine Coon Rls were proposed for red blood cell count, mean corpuscular volume, mean corpuscular haemoglobin concentration, reticulocyte count and percentage. Haematocrit was higher in male cats (mean HCT $42.9 \%$ vs $41 \%$ in females; $P=0.001$ ) and in heavier cats $(P=0.003$; slope 1.0, regression equation $\mathrm{HCT}=35.1+1.0 \times$ weight). New biochemical Rls were proposed for urea, aspartate aminotransferase, $\gamma$-glutamyl transpeptidase (GGT), alkaline phosphatase, total protein and albumin in Maine Coons. Females had higher GGT (median GGT value in females 4.0 vs 3.0 in males; $P=0.011$ ) and albumin values (mean albumin value 3.3 in females vs 3.1 in males; $P=0.013$ ).

Conclusions and relevance Currently published Rls for some haematological and biochemical parameters are not appropriate for use in adult Maine Coon cats. A breed-specific variation could be a plausible explanation for the new haematological and serum biochemical analytes proposed in this study. Breed-specific RIs for Maine Coon cats will help prevent misinterpretation of laboratory results in diagnosis and in the selection of ideal blood donors.
\end{abstract}

Accepted: 17 December 2014

\section{Introduction}

The Maine Coon cat breed was created in the USA in the early 1900s. This breed is genetically predisposed to hypertrophic cardiomyopathy $(\mathrm{HCM}),{ }^{1}$ is at increased risk for cutaneous haemangiosarcoma, ${ }^{2}$ has a high incidence of hip dysplasia, ${ }^{3}$ and it is also suspected to be at increased risk for haemophilia owing to deficiencies in factor I and factor XI. ${ }^{4}$ As these are large cats and live mostly indoors, they are often evaluated as potential blood donors in feline blood donor programmes. ${ }^{5}$

Recent studies have highlighted the importance of breed-related differences between haematological and biochemical values, ${ }^{6,7}$ raising the possibility that interbreed differences could be important in the interpretation of the results of certain diagnostic tests.
1Veterinary Transfusion Unit (REV), Department of Health, Animal Science and Food Safety (VESPA), University of Milan, Milan, Italy ${ }^{2}$ Veterinary Transfusion Unit (EMOVET-UNIPG), Department of Veterinary Clinical Science, University of Perugia, Perugia, Italy

Part of this work has been presented as a poster at the 16th European Society of Veterinary Clinical Pathology (ESVCP) Annual Congress, October 1-4, 2014, Milan, Italy.

\section{Corresponding authors:}

Eva Spada DVM, PhD, Veterinary Transfusion Unit (REV), Department of Health, Animal Science and Food Safety (VESPA), University of Milan, Via G. Celoria 10, 20133 Milan, Italy Email: eva.spada@unimi.it

Arianna Miglio DVM, PhD, Veterinary Transfusion Unit (EMOVETUNIPG), Department of Veterinary Clinical Science, University of Perugia, Via San Costanzo, 4, 06124 Perugia, Italy

Email: miglioarianna@libero.it 
For the reasons cited above, and because of the breed's worldwide popularity, it is important for clinicians to recognise the potential clinicopathological breed-specific features of Maine Coons. Haematological and biochemical profiles are used routinely to screen cats for disease and to monitor clinical progression, and breed-related differences in haematological and biochemical parameters may have an impact on clinical decision making. In addition, with recent advances in feline transfusion medicine and the increasing numbers of cats being evaluated as potential blood donors, it is important to recognise breed-specific clinicopathological features in order to make appropriate selections of blood donors following screening. The feline blood donor databank, recently created in Italy, includes results from a large number of healthy adult animals, and provides an excellent source for retrospective determination of haematological and biochemical reference intervals (RIs).

The aims of this retrospective study were three-fold: (1) to derive Maine Coon haematological and biochemical RIs from a large population of client-owned adult healthy blood donors; (2) to validate (or reject) published RIs for the general feline population in this breed; and (3) to evaluate the effects of age, sex and weight on the haematological and biochemical results.

\section{Materials and methods}

\section{Sample population}

The reference population consisted of Maine Coon cats sampled for voluntary blood donations at the Veterinary Transfusion Unit of the University of Perugia (EMOVETUNIPG) between January 2011 and January 2014. Written consent to draw and use blood for this study was obtained from all owners of participating cats.

Although cats included in this study were active blood donors and donate blood annually, the data used to calculate the RI in this study was that from the first evaluation of the cats when they were presented for initial screening. For pre-donation screening, cats were fasted for $12 \mathrm{~h}$ prior to blood collection.

Inclusion criteria for potential blood donor cats were as follows: healthy cats aged between 1 and 8 years; indoor lifestyle; weight $>5 \mathrm{~kg}$; up-to-date history for vaccination for feline herpesvirus, calicivirus and parvovirus, and prophylactic treatment for ecto-and endoparasites; to have received no treatments in the month before screening; and to have never received a blood transfusion.

Cats were considered healthy based on medical history, physical examination, cardiological evaluation (negative for $\mathrm{HCM}$ with conventional echocardiographic and Doppler examination, and negative for MyBPC3A31P mutation based on direct DNA sequence analysis), ${ }^{8}$ and have normal systolic, mean and diastolic arterial blood pressure (measured with a high definition oscillometric instrument, using the coccygeal artery at the tail base of each cat).

Following the Italian guidelines on veterinary transfusion medicine, ${ }^{9}$ all blood donor cats were tested and found to be seronegative for feline immunodeficiency virus (FIV; enzyme-linked immunosorbent assay [ELISA] test), feline leukaemia virus (FeLV; ELISA test) and feline coronavirus infection (FCoV; anti-FCoV serology by indirect immunofluorescent test). Blood smears were negative for haemotropic Mycoplasma, and faecal parasitology examination was negative for enteric parasites. All cats were blood typed (immunochromatographic test).

\section{Sample collection}

A single operator collected samples from all cats by jugular venepuncture. Samples were collected using $23 \mathrm{G}$ needles and $3 \mathrm{ml}$ syringes, and immediately transferred to K3-ethylenediaminetetraacetic acid (Terumo) and serum gel collection tubes (BD Vacutainer).

The aggregate reticulocyte count was manually performed as follows: 1 part blood was mixed with 1 part new methylene blue in a small test tube and incubated at room temperature. After 15 mins, the contents were mixed and a small drop of the mixture was placed on a clean glass slide and a thin smear prepared.

Each gel tube was centrifuged at $1500 \mathrm{~g}$ for 5 mins within 10 mins of collection, and the serum supernatant was harvested and immediately analysed.

\section{Haematological analyses}

A complete blood count was obtained from each sample within $1 \mathrm{~h}$ of blood collection, using a haematology analyser (Sysmex XT-1800iV; Sysmex) that combines impedance and optical methods, and for which specific veterinary software has been developed (software v. 9). The Sysmex haematological analyser has already been validated for use in feline blood samples.10,11 Measured variables included red blood cell (RBC) count by impedance method (RBC-I), haemoglobin concentration, haematocrit (HCT), mean corpuscular volume (MCV), mean corpuscular haemoglobin $(\mathrm{MCH}), \mathrm{MCH}$ concentration (MCHC), platelet count by impedance method and white blood cell (WBC), neutrophil, lymphocyte, monocyte and eosinophil counts by laser method. Basophil counts were not recorded as they have been reported to be unreliable when measured in feline blood analysed by Sysmex. ${ }^{11}$

To minimise random and systematic errors that have been associated with automated differential counts, including those reported by the Sysmex instruments, ${ }^{11}$ we opted to include only automated differential counts that had been validated by microscopic blood smear examination. For all samples, air-dried Wright-Giemsastained blood films were made immediately after 
collection and evaluated by the same veterinary clinical pathologist to validate automated WBC counts and to perform morphological assessment of blood cells. Differential WBC counts were performed manually by counting 100 nucleated cells per smear. The presence or absence of platelet clumping was noted.

All analyses were performed following the manufacturer's recommendations and quality controls for assay conditions and instrumentation; a daily maintenance self-test was always carried out before performing any assay.

The number of reticulocytes was counted in oil immersion magnification $(\times 1000)$ in five arbitrary fields of 200 RBCs. Percentage of aggregate reticulocytes was determined by number of reticulocytes counted divided by 10.12

\section{Biochemical analyses}

Selected biochemical analytes were analysed on an automated spectrophotometer clinical wet chemistry analyser (Hitachi 904; Boehringer Mannheim) with reagents provided by Roche Diagnostics. The analytes measured were urea (Kinetik ultraviolet [UV] method), creatinine (Jaffè kinetic method), alanine aminotransferase (ALT) and aspartate aminotransferase (AST) (kinetic International Federation of Clinical Chemistry [IFCC] method), $\gamma$-glutamyl transpeptidase (GGT) (kinetic IFCC method), alkaline phosphatase (ALP; kinetic IFCC method), albumin (bromocresol method), total protein (TP; biuret method), phosphate (molibdate UV method).

Prior to each sample run, analysers were calibrated according to the manufacturers' instructions and control samples were analysed. Control samples consisted of various commercially available standards with values within the manufacturers' recommended limits. Grossly haemolysed or lipaemic samples were discarded.

\section{Statistical analysis}

A non-parametric method was used to determine the reference limits (with the lower reference limit being the 2.5th percentile and the upper reference limit being the 97.5th percentile) and their $90 \%$ confidence intervals (CIs) using the bootstrap method with Reference Value Advisor (freeware v 2.1; http://www.biostat.envt.fr/ spip/spip.php?article63) set of macroinstructions. ${ }^{13,14}$

The software performs the following computations recommended by the International Federation of Clinical Chemistry - Clinical and Laboratory Standards Institute, ${ }^{15}$ and American Society of Veterinary Clinical Pathology (ASVCP) RI guidelines: ${ }^{16}$ descriptive statistics (eg, mean, median, SD, minimum and maximum values); tests of normality (Anderson-Darling with histograms, and $\mathrm{Q}-\mathrm{Q}$ plots and Box-Cox transformation); and outlier analysis. Both Dixon-Reed and Tukey tests were used, and outliers classified as 'suspected' were retained, as recommended by the ASVCP guidelines, ${ }^{16}$ while extreme outliers were removed from the analysis. The non-parametric approach requires no assumptions regarding the distribution of data, and it is recommended by the Clinical Laboratory Standards Institute (CLSI) when sufficient number of reference values can be obtained. ${ }^{15}$ This software was also used to determine how continuous covariates (weight and age) were linked to RI using a regression and analysis of variance model.

To calculate RIs for Maine Coons, after removal of data that, according to the Tukey rule, behave as extreme outliers interpretable as aberrant observations, 20 Maine Coon reference individuals were randomly selected by use of a random number generator program (RAND function of Microsoft Excel), and their results were compared with the haematological RIs for the general feline population obtained with Sysmex $\mathrm{XT}-2000 \mathrm{iV},{ }^{17}$ and plasma biochemical RIs for Maine Coons and serum biochemical RIs for the general feline population published in two textbooks.7,18,19 The transference study followed the validation procedure recommended by CLSI C28A3c and ASCVP RI guidelines. ${ }^{15,16}$ RIs were considered validated if $\leqslant 10 \%(n=0-2)$ of the Maine Coon values fell outside the published reference limits. RIs were rejected if $>25 \%$ of the reference values $(n \geqslant 5)$ fell outside the reference limits. If $>10 \%$ but $<25 \%$ of the reference values $(n=3-4)$ fell outside the reference limits, a new set of 20 animals was randomly selected and their reference values compared with the RIs. In the new setup, if $\leqslant 10 \%$ of the samples fell outside the RIs, then the laboratory RI was considered validated; otherwise, it was rejected.

The correlation between sex (male and female) and haematological and biochemical parameters was assessed using a general linear model. A significant age effect $(P<0.05)$ was further studied by regression analysis.

All calculations were performed with Microsoft Excel 2010 and with MedCalc software (version 12.7.0.0; Mariakerke).

\section{Results}

In total, from a reference population of 93 Maine Coon blood donors, data from 81 healthy cats from four catteries were available for the final determination of haematological and biochemical RIs. No cats were included more than once in the study population. The reference sample group was composed of 42 intact female and 39 intact male cats, aged from 1-8 years (mean \pm SD $4.4 \pm 2.0$ years, median 4.0 years) and weighing between 4.9 and $8.5 \mathrm{~kg}$ (mean $\pm \mathrm{SD} 6.7 \pm 0.9 \mathrm{~kg}$, median 6.7 years). All cats were type A blood group.

Age distribution was independent of sex and weight. Males were heavier (mean $7.3 \mathrm{~kg}$, range 5.8-8.5) than females (median $6.1 \mathrm{~kg}$, range 4.9-7.4) $(P<0.001)$. 
Table 1 New haematological reference intervals (RIs) established in 81 adult Maine Coon blood donor cats reported according to the Clinical and Laboratory Standards Institute and American Society of Veterinary Clinical Pathology guidelines. . $^{15,16}$ In three blood samples, leukocyte differential count was obtained by microscopic blood smear examination

\begin{tabular}{|c|c|c|c|c|c|c|c|c|}
\hline Analyte & Mean & $\mathrm{SD}$ & Median & Min & Max & $\mathrm{RI}$ & $\begin{array}{l}\text { Lower } \\
\text { reference } \\
\text { limit 90\% Cl }\end{array}$ & $\begin{array}{l}\text { Upper } \\
\text { reference } \\
\text { limit } 90 \% \mathrm{Cl}\end{array}$ \\
\hline RBC-I $\left(10^{12} / 1\right)$ & 6.4 & 0.9 & 6.4 & 3.96 & 8.65 & $4.6-8.5$ (1S) & $4.0-5.0$ & $8.2-8.7$ \\
\hline Haemoglobin $(\mathrm{g} / \mathrm{l})$ & 148 & 8 & 147 & 128 & 171 & $133-163$ & $128-136$ & $161-171$ \\
\hline Haematocrit (I/I) & 0.41 & 0.30 & 0.42 & 0.37 & 0.48 & $0.37-0.48$ & $0.37-0.38$ & $0.46-0.48$ \\
\hline $\operatorname{MCV}(f I)$ & 48.5 & 4.3 & 48.0 & 39.0 & 61.0 & $41.1-59.0(2 S)$ & $39.0-42.0$ & $55.9-61.0$ \\
\hline MCH (pg/cell) & 13.6 & 0.7 & 13.5 & 12.0 & 15.8 & $12.2-15.8(5 \mathrm{~S}, 2 \mathrm{R})$ & $12.0-12.5$ & $15.2-15.8$ \\
\hline $\mathrm{MCHC}(\mathrm{g} / \mathrm{l})$ & 33.7 & 2.5 & 33.3 & 28.5 & 40.2 & $29.1-40.0(5 S)$ & $28.5-30.0$ & $38.6-40.2$ \\
\hline WBC (109/I) & 9.7 & 2.4 & 9.4 & 5.3 & 16.7 & $5.7-15.6$ (1S) & $5.3-5.9$ & $13.5-16.7$ \\
\hline Neutrophils $\left(10^{9} / 1\right)$ & 6.3 & 1.7 & 6.2 & 3.3 & 11.4 & $3.4-10.8$ & $3.3-3.9$ & $8.9-11.4$ \\
\hline Eosinophils (109/l) & 0.3 & 0.2 & 0.3 & 0.0 & 0.7 & $0.0-0.7$ & $0.0-0.1$ & $0.6-0.7$ \\
\hline Lymphocytes (109/l) & 2.5 & 0.8 & 2.3 & 0.8 & 5.6 & $1.1-4.6(1 S)$ & $0.8-1.5$ & $4.0-5.6$ \\
\hline Monocytes (10\%/l) & 0.5 & 0.2 & 0.5 & 0.1 & 1.1 & $0.2-1.0(2 \mathrm{~S}, 1 \mathrm{R})$ & $0.1-0.2$ & $0.8-1.1$ \\
\hline PLT-I (109/I) & 200.0 & 76.4 & 178.0 & 58.0 & 398.0 & $85.0-385.0(3 S)$ & $58.0-93.0$ & $351.0-398.0$ \\
\hline Reticulocytes (\%)* & 1.4 & 0.8 & 1.3 & 0.1 & 4.6 & $0.2-3.3(1 \mathrm{~S})$ & $0.1-0.5$ & $2.9-4.6$ \\
\hline Reticulocytes $\left(10^{9} / 1\right)^{\star}$ & 88.1 & 55.6 & 73.3 & 6.7 & 295.8 & $11.3-250.0(4 S)$ & $6.7-28.9$ & $200.6-295.8$ \\
\hline
\end{tabular}

$(n S)$ indicates the number of suspected outliers present according to Tukey, (nR) indicates the number of far outliers identified according to Tukey or Dixon and removed. The sample size for each analyte is large enough to compute the non-parametric RI. The confidence intervals (Cls) of the limits of the non-parametric RI were determined using a bootstrap method

*Manual count, only aggregate reticulocytes counted

$\mathrm{RBC}-\mathrm{I}$ = red blood cell count by impedance measurement; $\mathrm{MCV}=$ mean cell volume; $\mathrm{MCH}=$ mean corpuscular haemoglobin; $\mathrm{MCHC}=\mathrm{mean}$ corpuscular haemoglobin concentration; WBC = white blood cells; PLT-I = platelet count by impedance measurement

Results for haematological and biochemical data from Maine Coons (mean, SD, median, minimum, maximum, proposed RI for each parameter, lower and upper reference limit 90\% CI) are summarised in Tables 1 and 2, respectively. All but three automated differential counts had been validated by microscopic blood smear examination. Platelet clumping was found in 48/81 (59.2\%) samples. Platelet aggregation scores were not recorded. No samples were discarded for reasons of gross haemolysis or lipaemia.

Haematological data from Maine Coons were compared with that provided by Granat et $a{ }^{17}{ }^{17}$ for the Sysmex XT-2000iV analyser (ie, for the general feline population), and the percentage of the Maine Coon population that was outside the non-breed-specific RIs is reported in Table 3. Based on comparison with published feline RIs, new Maine Coons RIs are proposed for RBC count, MCV, MCHC, and reticulocyte count and percentage.

Similarly, biochemical data from the Maine Coons were compared with RIs provided by Reynolds et $\mathrm{al}^{7}$ for Maine Coon plasma biochemical RIs determined using dry chemistry, and with RIs for the general feline population on serum samples reported in two textbooks. ${ }^{18,19}$ The percentage of the Maine Coon population that was outside non-breed-specific RIs and the Maine Coon-specific
RIs is reported in Tables 4 and 5, respectively. Based on comparison with published RIs for the general feline population, new biochemical RIs were proposed for Maine Coons for urea, AST, GGT, ALP, TP and albumin.

There was a positive correlation between both body weight and sex and HCT value. Male cats (mean HCT $42.9 \%$ vs $41 \%$ in female; $P=0.001$ ) and heavier cats $(P=0.003$; regression equation $\mathrm{HCT}=35.1+1.0 \times$ weight) having higher HCT. The only positive correlation in the biochemical data was between sex and both GGT and albumin value, with females having higher GGT (median GGT value in female 4.0 vs 3.0 in male; $P=0.011$ ) and albumin (mean albumin value 3.3 in female vs 3.1 in male; $P=0.013$ ).

\section{Discussion}

Differences between haematological and biochemical values in different feline breeds have been recently reported, $, 7,20$ raising the possibility that interbreed differences may have a significant impact on the interpretation of certain diagnostic test results.

In this study we evaluated a blood donor database to calculate specific haematological and biochemical RIs for Maine Coons. This breed is a popular breed prone to important diseases requiring veterinary attention and eventual blood testing (eg, HCM). ${ }^{1}$ In addition, Maine 
Table 2 Reference intervals (RIs) for selected serum biochemical parameters in 81 healthy owned blood donor adult Maine Coon cats using wet chemistry analyser (Hitachi 904)

\begin{tabular}{|c|c|c|c|c|c|c|c|c|}
\hline Analyte & Mean & SD & Median & Min & Max & $\mathrm{RI}$ & $\begin{array}{l}\text { Lower } \\
\text { reference } \\
\text { limit } 90 \% \mathrm{Cl}\end{array}$ & $\begin{array}{l}\text { Upper } \\
\text { reference } \\
\text { limit } 90 \% \mathrm{Cl}\end{array}$ \\
\hline Creatinine $(\mu \mathrm{mol} / \mathrm{l})$ & 97.2 & 35.4 & 106.1 & 30.1 & 154.7 & $35.4-150.3$ & $26.5-44.2$ & $150.3-159.1$ \\
\hline Urea (mmol/l) & 15.1 & 4.9 & 15.4 & 4.3 & 27.8 & $5.8-24.2$ & $4.3-6.9$ & $21.7-27.9$ \\
\hline $\operatorname{ALT}(\mathrm{U} / \mathrm{l})$ & 30.1 & 13.4 & 29.0 & 5.0 & 75.0 & $8.1-72.9(4 S)$ & $5.0-10.0$ & $46.0-75.0$ \\
\hline AST (U/I) & 21.9 & 7.4 & 20.0 & 9.0 & 45.0 & $10.1-38.9(1 S)$ & $9.0-11.1$ & $33.0-45.0$ \\
\hline GGT (U/I) & 3.6 & 2.2 & 3.0 & 0.0 & 9.0 & $0.0-8.0$ & $0.0-0.0$ & $8.0-9.0$ \\
\hline ALP $(U / I)$ & 55.2 & 28.3 & 54.0 & 12.0 & 165.0 & $14.1-110.0(1 S)$ & $12.0-17.0$ & $99.5-165.0$ \\
\hline Phosphate $(\mathrm{mmol} / \mathrm{l})$ & 1.5 & 0.2 & 1.5 & 0.9 & 1.9 & $0.9-1.8$ & $0.9-1.1$ & $1.7-1.9$ \\
\hline Total protein (g/l) & 70 & 7 & 70 & 51 & 89 & $51-87(4 S)$ & $51-57$ & 80-89 \\
\hline Albumin (g/l) & 32.0 & 4.0 & 32.0 & 23.4 & 41.8 & $24.0-41.0$ & $23.0-27.0$ & $38.0-42.0$ \\
\hline
\end{tabular}

(nS) indicates the number of suspected outliers present according to Tukey. The sample size for each analyte is large enough to compute the non-parametric reference interval. The confidence intervals (Cls) of the limits of the non-parametric reference interval were determined using a bootstrap method

$\mathrm{ALT}=$ alanine aminotransferase; $\mathrm{AST}=$ aspartate aminotransferase; $\mathrm{GGT}=\gamma$-glutamyl transpeptidase; $\mathrm{ALP}=$ alkaline phosphatase

Table 3 Published feline reference intervals (RIs) for the Sysmex XT-2000iV analyser, ${ }^{17}$ and the new adult Maine Coonspecific Rls (Sysmex XT-1800iV)

\begin{tabular}{|c|c|c|c|}
\hline Analyte & Published RI ${ }^{17}$ & Outside RI (\%) & Maine Coon RI \\
\hline RBC-I $\left(10^{12} /\right)$ & $6.7-11.2$ & 65 & $4.6-8.5$ \\
\hline Haemoglobin $(\mathrm{g} / \mathrm{l})$ & $99.1-165.9$ & 0 & $133.0-163.0$ \\
\hline Haematocrit (I/I) & $0.29-0.51$ & 0 & $0.37-0.48$ \\
\hline $\operatorname{MCV}(f \mid)$ & $34.4-49.3$ & 30 & $41.1-59.0$ \\
\hline MCH (pg/cell) & $12.3-16.4$ & 0 & $12.2-15.8$ \\
\hline $\mathrm{MCHC}(\mathrm{g} / \mathrm{dl})$ & $32.5-39.2$ & 35 & $29.1-40.0$ \\
\hline WBC (109/l) & $4.0-15.2$ & 0 & $5.7-15.6$ \\
\hline Neutrophils $\left(10^{9} / 1\right)$ & $1.7-8.8$ & 0 & $3.4-10.8$ \\
\hline Eosinophils (109/) & $0.1-1.2$ & 0 & $0.0-0.7$ \\
\hline Lymphocytes $\left(10^{9} / l\right)$ & $1.2-10.2$ & 0 & $1.1-4.6$ \\
\hline Monocytes (109/l) & $0.1-0.6$ & 10 & $0.2-1.0$ \\
\hline PLT-I (109/I) & $33-516$ & 0 & $85-385$ \\
\hline Reticulocytes (\%) & $0.2-1.1^{*}$ & 50 & $0.2-3.3^{\dagger}$ \\
\hline Reticulocytes (109/l) & $19.1-107.1^{\star}$ & 35 & $11.3-250.0^{\dagger}$ \\
\hline
\end{tabular}

RIs were considered validated if $\leqslant 10 \%(n=0-2)$ of the Maine Coon values fell outside the published reference limits. Rls were rejected if $>25 \%$ of the reference values $(n \geqslant 5)$ fell outside the reference limits. If $>10 \%$ but $<25 \%$ of the reference values $(n=3-4)$ fell outside the reference limits, a new set of 20 animals was randomly selected and their reference values compared with the Rls. In the new set-up, if $\leqslant 10 \%$ of the samples fell outside the RIs, then the laboratory RI was considered validated; otherwise, it was rejected. When the published RI was rejected, a new RI, reported in bold, was established following the Clinical and Laboratory Standards Institute and American Society of Veterinary Clinical Pathology guidelines ${ }^{15,16}$

*Aggregate and punctate reticulocytes count, tManual count, only aggregate reticulocytes counted

$\mathrm{RBC}-\mathrm{I}=$ red blood cell count by impedance measurement; $\mathrm{MCV}=$ mean cell volume; $\mathrm{MCH}=$ mean corpuscular haemoglobin; $\mathrm{MCHC}=$ mean corpuscular haemoglobin concentration; WBC $=$ white blood cells; PLT-I = platelet count by impedance measurement

Coon cats are large cats, most of which have an indoor lifestyle, with a good nature, and these qualities make them ideal candidates for blood donation.

When haematological RIs for Maine Coons are compared with the general feline population measured on a Sysmex XT-2000iV, ${ }^{17}$ the majority are similar. However, there were significant variations in results for some parameters; notably, Maine Coons had a lower limit for
RBC count than the general feline population, a higher upper limit for MCV and a lower limit for MCHC. In addition, Maine Coon cats showed a higher aggregate reticulocyte count (two- to three-fold that of the general feline population). Together these haematological data suggest a macrocytic hypochromic anaemia (regenerative anaemia with large, young RBCs with low haemaglobin concentrations). ${ }^{21}$ However, all cats in this study 
Table 4 Feline reference intervals (RIs) for serum biochemical parameters for the general feline population published in two textbooks ${ }^{18,19}$

\begin{tabular}{|llllll|}
\hline Analyte & Kaneko et al ${ }^{18}$ & Outsider RI (\%) & Tvedten $^{19}$ & Outsider RI (\%) & Maine Coon RI \\
\hline Creatinine $(\mu \mathrm{mol} / \mathrm{l})$ & $70.7-159$ & 50 & $61.9-194.5$ & $15(0)$ & $35.4-150.3$ \\
Urea $(\mathrm{mmol} / \mathrm{l})$ & $3.33-5.00$ & 100 & $6.43-14.64$ & 55 & $\mathbf{5 . 8 - 2 4 . 2}$ \\
ALT $(\mathrm{U} / \mathrm{l})$ & $6-83$ & 0 & $23-109$ & 30 & $8.1-72.9$ \\
AST $(\mathrm{U} / \mathrm{l})$ & $26-43$ & 90 & $14-41$ & $20(15)$ & $\mathbf{1 0 . 1 - 3 8 . 9}$ \\
GGT $(\mathrm{U} / \mathrm{l})$ & $1.3-5.1$ & 35 & $1-3$ & 45 & $\mathbf{0 . 0 - 8 . 0}$ \\
ALP $(\mathrm{U} / \mathrm{l})$ & $25-93$ & $15(30)$ & $4-81$ & $15(25)$ & $\mathbf{1 4 . 1 - 1 1 0}$ \\
Phosphorus $(\mathrm{mmol} / \mathrm{l})$ & $1.45-2.62$ & 45 & $0.94-2.68$ & 5 & $0.9-1.8$ \\
Total protein $(\mathrm{g} / \mathrm{l})$ & $54.0-78.0$ & $20(15)$ & $55.0-77.0$ & $20(15)$ & $\mathbf{5 1 . 0 - 8 7 . 0}$ \\
Albumin $(\mathrm{g} / \mathrm{l})$ & $21.0-33.0$ & 30 & $30.0-46.0$ & 40 & $\mathbf{2 4 . 0 - 4 1 . 0}$
\end{tabular}

RIs were considered validated if $\leqslant 10 \%(n=0-2)$ of the Maine Coon values fell outside the published reference limits. RIs were rejected if $>25 \%$ of the reference values $(n \geqslant 5)$ fell outside the reference limits. If $>10 \%$ but $<25 \%$ of the reference values $(n=3-4)$ fell outside the reference limits, a new set of 20 animals was randomly selected and their reference values compared with the RIs. In the new set-up, if $\leqslant 10 \%$ of the samples fell outside the RIs, then the laboratory RI was considered validated; otherwise, it was rejected. When the published RI was rejected, a new RI, reported in bold, was established following the Clinical and Laboratory Standards Institute and American Society of Veterinary Clinical Pathology guidelines ${ }^{15,16}$

$\mathrm{ALT}=$ alanine aminotransferase; AST $=$ aspartate aminotransferase; GGT $=\gamma$-glutamyl transpeptidase; ALP $=$ alkaline phosphatase

Table 5 Published feline reference intervals (Rls) for plasma biochemical parameters (measured using dry reagents) in 139 healthy Maine Coon cats ${ }^{7}$

\begin{tabular}{|llll|}
\hline Analyte & Published Maine Coon Rls & Outsider RI (\%) & Maine Coon RI (this study) \\
\hline Creatinine $(\mu \mathrm{mol} / \mathrm{l})$ & $77-192$ & 55 & $\mathbf{3 5 . 4 - 1 5 0 . 3}$ \\
Urea $(\mathrm{mmol} / \mathrm{l})$ & $6.1-11.2$ & 85 & $\mathbf{5 . 8 - 2 4 . 2}$ \\
ALT $(\mathrm{U} / \mathrm{l})$ & $24-103$ & 35 & $\mathbf{8 . 1 - 7 2 . 9}$ \\
ALP $(\mathrm{U} / \mathrm{l})$ & $28-107$ & 10 & $14.1-110.0$ \\
Phosphate $(\mathrm{mmol} / \mathrm{l})$ & $0.90-2.79$ & 5 & $0.9-1.8$ \\
Total protein $(\mathrm{g} / \mathrm{l})$ & $59-85$ & 0 & $51-87$ \\
Albumin $(\mathrm{g} / \mathrm{dl})$ & $2.3-3.6$ & 0 & $2.4-4.1$ \\
\hline
\end{tabular}

RIs were considered validated if $\leqslant 10 \%(n=0-2)$ of the Maine Coon values fell outside the published reference limits. RIs were rejected if $>25 \%$ of the reference values $(n \geqslant 5)$ fell outside the reference limits. If $>10 \%$ but $<25 \%$ of the reference values $(n=3-4)$ fell outside the reference limits, a new set of 20 animals was randomly selected and their reference values compared with the RIs. In the new set-up, if $\leqslant 10 \%$ of the samples fell outside the RIs, then the laboratory RI was considered validated; otherwise, it was rejected. When the published RI was rejected, a new RI, reported in bold, was established following the Clinical and Laboratory Standards Institute and American Society of Veterinary Clinical Pathology guidelines ${ }^{15,16}$

$\mathrm{ALT}=$ alanine aminotransferase; ALP = alkaline phosphatase

were clinically healthy, negative for the most important viral and bacterial infections that cause feline anaemia (ie, FIV, FeLV, haemoplasma infection) and were blood sampled before entering a blood donation programme. Therefore, these haematological characteristics appear to be normal for this breed. These apparently physiological variations need to be considered when evaluating Maine Coons as potential blood donors; otherwise, healthy cats may well be excluded from blood donor programmes. As expected, heavier cats (primarily male cats) have higher HT and are therefore preferred for inclusion in blood donor programmes.

It would be interesting to compare the RIs established in this study with those published in the veterinary literature for this feline breed. A recent publication includes RIs in Maine Coon cats for biochemical parameters. ${ }^{7}$
Although the RIs in that study were calculated from a larger population $(n=139)$, they were from plasma samples analysed using a dry reagent analyser. In addition, the tested population also included kittens and cats older than 10 years. This may explain why the previously published Maine Coon RIs were rejected for urea, creatinine and ALT, which can all be affected by age and differences in sample type and analyser used.

Different RIs were identified for Maine Coon cats for 6/9 serum biochemical variables tested, that is for urea, AST, GGT, ALP, total protein and albumin when compared with RIs for the general feline population in two textbooks. ${ }^{18,19}$ However, direct comparison with the RIs in textbooks poses some questions. For most of the published RIs in cats, there is limited information regarding the reference sample group, the methodology of 
instruments used (wet vs dry chemistry, different single parameters method) and the methods of calculation of the RIs. Moreover, the reference population may be different from that of this study (eg, our RIs have been generated from intact cats, younger than 8 years, while the general hospital population usually comprises neutered and/or aged animals).

Our large sample size population provided a representative sample of the population of adult healthy Maine Coon cats and enabled the use of non-parametric methods for establishing RIs, as currently recommended. ${ }^{15,16}$ However, our population may not be representative of the entire Maine Coon feline patient population as there were no very young $(<1$ year) cats or cats older than 8 years included. However, the breed-specific RIs found in this study provide a useful tool for the clinical interpretation of haematological profiles in Maine Coons. These RIs may be useful to other laboratories using similar instrumentation and/or methods of analysis.

It is likely that some of the differences in RIs reported in this study (ie, urea, GGT, ALP and TP), are genuine breed-related differences and highlight the need for new RIs in Maine Coons. As in Abyssinians, Siberians and Holly Birman cats, ${ }^{6}$ the upper limit for total protein and albumin appears higher than that in the general population. Race-related differences in serum proteins have also been described in humans. ${ }^{22}$

The upper reference limits for ALP and GGT activities were significantly higher in Maine Coon cats compared with standard laboratory RIs. Serum ALP activity may be increased in young, growing cats, in skeletal, endocrine or hepatobiliary disease, and by drug administration, while GGT usually is increased owing to biliary disease. ${ }^{18,19}$ All cats included in the study were clinically healthy adults and none were receiving medication. Other indicators of liver disease such as increased ALT activity were not detected. Even though the total bilirubin concentration was not measured and ultrasonographic examination of the hepatobiliary system was not performed, it is unlikely the higher levels of these analytes indicated hepatobiliary problems; rather, they may be a physiologic feature of Maine Coon cats.

As already reported in a previous study in Norwegian Forest cats, ${ }^{6}$ Maine Coon cats also showed an increased ALP activity with respect to the general feline population. As kittens aged $<1$ year of age were excluded from this study, this finding cannot be due to bone remodelling typical of young animals. ${ }^{23,24}$ However, it is possible that larger cats, such as Maine Coons and Norwegian Forest cats, continue to have an active bone metabolism into young adulthood. This hypothesis could be addressed in future studies by evaluating the different ALP fractions in blood samples of Maine Coon cats. ${ }^{24}$

In this study, RIs for haematological and biochemical parameters in Maine Coons were determined using an indirect sampling method, as reference individuals were selected from a large database of blood donor cats. This method, previously demonstrated in veterinary medicine, ${ }^{25}$ has several advantages compared with the traditional method of determining RIs (selecting reference individuals from a sample population), including its relative simplicity, low cost and capacity to include a large number of reference individuals. Limitations of this method include the inability to control some pre-analytical and analytical factors, such as specimen collection and handling, and analysis of specimens using different analysers. We attempted to minimise these disadvantages by using data from one university transfusion unit (EMOVETUNIPG), where blood collection and analysis were performed by the same operator in a standardised manner.

When analysing a blood donor database retrospectively the risk of including values from unhealthy individuals should be less than that from using a prospective study as the potential blood donor cats were clinically and haematologically monitored at least annually (every time they donated blood) and were examined more frequently and extensively to determine health status and suitability for blood donation (eg, full cardiological evaluation and infectious diseases screening). It is essential that Maine Coon cats undergo a full cardiological evaluation before being declared healthy subjects, as one study demonstrated that Maine Coon cats with HCM were more likely $(P<0.05)$ to have a normal auscultation than other breeds. ${ }^{26}$

One limitation of our sample reference population is that the cats included in this study originated from just four catteries and from a single study site, which may limit the genetic diversity of the reference population.

\section{Conclusions}

We found significant differences in Maine Coons from the general feline population in four haematological and six serum biochemical parameters, for which a breedspecific variation could be a plausible explanation. Breed-specific RIs for Maine Coon cats will help prevent misinterpretation of laboratory results in diagnosis and in the selection of suitable blood donors. Further investigations are necessary to understand the mechanisms underlying these differences.

Conflict of interest The authors do not have any potential conflicts of interest to declare.

Funding This research received no specific grant from any funding agency in the public, commercial or not-for-profit sectors.

\section{References}

1 Meurs KM, Sanchez X, David RM, et al. A cardiac myosin binding protein $\mathrm{C}$ mutation in the Maine coon cat with familial hypertrophic cardiomyopathy. Hum Mol Genet 2005; 14: 3587-3593. 
2 Goldschmidt MH and Mcmanus P. Dermatopathology. Skin tumors of the dog and cat. http:/ / cal.vet.upenn.edu/ projects/derm/index.html (2000, accessed July 7, 2014).

3 Keller GG, Reed AL, Lattimer JC, et al. Hip dysplasia: a feline population study. Vet Radiol Ultrasound 1999; 40: 460-464.

4 Brown R. Haemophilia in Maine Coon cats. Vet Rec 2008; 163: 667.

5 Spada E, Miglio A, Proverbio D, et al. Signalment and blood types in cats being evaluated as blood donors at two Italian university blood banks. Vet Med Int 2014; 2014: 704836

6 Paltrinieri S, Ibba F and Rossi G. Haematological and biochemical reference intervals of four feline breeds. J Feline Med Surg 2014; 16: 125-136.

7 Reynolds BS, Concordet D, Germain CA, et al. Breed dependency of reference intervals for plasma biochemical values in cats. J Vet Intern Med 2010; 24: 809-818.

8 Longeri M, Ferrari P, Knafelz P, et al. Myosin-binding protein C DNA variants in domestic cats (A31P, A74T, R820W) and their association with hypertrophic cardiomyopathy. J Vet Intern Med 2013; 27: 275-285.

9 Italian Health Minister. Guide Line relating to the exercise of the health activity concerning the transfusion medicine in the veterinary field. http://www.salute.gov.it/ imgs/C_17_pubblicazioni_852_allegato.pdf (2007, accessed September 15, 2014)

10 Lilliehöök I and Tvedten H. Validation of the Sysmex XT-2000iV hematology system for dogs cats and horses. I. Erythrocytes, platelets, and total leukocyte counts. Vet Clin Pathol 2009; 38: 163-174.

11 Lilliehöök I and Tveden H. Validation of the Sysmex XT$2000 \mathrm{iV}$ hematology system for dogs cats and horses. II. Differential leukocyte counts. Vet Clin Pathol 2009; 38: 175-182.

12 Cowgill ES, Neel JA and Grindem CB. Clinical application of reticulocyte counts in dogs and cats. Vet Clin North Am Small Anim Pract 2003; 33:1223-1244.

13 Geffré A, Concordet D, Braun JP, et al. Reference Value Advisor: a new freeware set of macroinstructions to calculate reference intervals with Microsoft Excel. Vet Clin Path 2011; 40: 107-112.

14 Geffré A, Friedrichs K, Harr K, et al. Reference values: a review. Vet Clin Path 2009; 38: 288-298.
15 CLSI. Defining, establishing, and verifying reference intervals in the clinical laboratory; approved guideline, document C28-A3c. 3rd ed. Wayne, PA: Clinical and Laboratory Standards Institute (CLSI), 2010.

16 Friedrichs KR, Harr KE, Freeman KP, et al. ASVCP reference interval guidelines: determination of de novo reference intervals in veterinary species and other related topics. Vet Clin Path 2012; 41: 441-453.

17 Granat F, Geffré A, Braun JP, et al. Feline reference intervals for the Sysmex XT-2000iV and the ProCyte DX haematology analysers in EDTA and CTDA blood specimens. J Feline Med Surg 2014; 16: 473-482.

18 Kaneko JJ, Harvey JW and Bruss ML. Appendix IX: blood analyte reference values in small and some laboratory animals. In: Kaneko JJ, Harvey JW and Bruss ML (eds). Clinical biochemistry of domestic animals. 6th ed. St Louis, MO: Elsevier, 2008, pp 889-895.

19 Tvedten H. Appendix II: listing of selected reference values. In: Willard MD and Tvedten $H$ (eds). Small animal clinical diagnosis by laboratory methods. 5 th ed. St Louis, MO: Elsevier Saunders, 2012, pp 399-402.

20 Gunn-Moore DA, Dodkin SJ and Sparkes AH. An unexpectedly high prevalence of azotaemia in Birman cats. J Feline Med Surg 2002; 4:165-166.

21 Tvedten $\mathrm{H}$. Laboratory and clinical diagnosis of anemia. In: Weiss DJ and Wardrop KJ (eds). Schalm's veterinary hematology. 6th ed. Aimes, IA: Wiley Blackwell, 2010, pp 152-161.

22 Kelley-Hedgepeth A, Lloyd-Jones DM, Colvin A, et al. Ethnic differences in C-reactive protein concentrations. Clin Chem 2008; 54: 1027-1037.

23 Fernandez NJ and Kidney BA. Alkaline phosphatase: beyond the liver. Vet Clin Path 2007; 36: 223-233.

24 Horney BS, Farmer AJ, MacKensie A, et al. Alkaline phosphatase isoenzymes in feline serum using an agarose gel alkaline phosphatase kit method. Can J Vet Res 1992; 56: 373-375.

25 Serra M, Freeman KP, Campora C, et al. Establishment of canine hematology reference intervals for the Sysmex XT$2000 \mathrm{iV}$ hematology analyzer using a blood donor database. Vet Clin Path 2012; 41: 207-215.

26 Trehiou-Sechi E, Tissier R, Gouni V, et al. Comparative echocardiographic and clinical features of hypertrophic cardiomyopathy in 5 breeds of cats: a retrospective analysis of 344 cases (2001-2011). J Vet Intern Med 2012; 26: 532-541. 